\title{
Topological phases in adiabatic and non-adiabatic driven systems
}

\author{
A. Gómez-León and G. Platero \\ Instituto de Ciencia de Materiales de Madrid (ICMM-CSIC), Cantoblanco, 28049 Madrid, Spain.
}

(Dated: March 3, 2018)

\begin{abstract}
In this work we study the geometrical and topological properties of non-equilibrium quantum systems driven by ac fields. We consider two tunnel coupled spin qubits driven by either spatially homogeneous or inhomogeneous ac fields. Our analysis is an extension of the classical model introduced by Berry with the addition of the spatial degree of freedom. We calculate the Berry and Aharonov-Anandan geometric phases, and demonstrate the influence of the different field parameters in the geometric properties. We also discuss the topological properties associated with the different driving regimes, and show that by tuning the different parameters one can induce topological phase transitions, even in the non-adiabatic regime.
\end{abstract}

PACS numbers:

Introduction: Geometric phases in quantum physics is a fundamental issue which has been addressed in the last decades. Since Berry's discovery of geometric phases in quantum systems [1], and their further applications to different branches of physics (e.g. condensed matter and optics), geometry, topology and nature seem to be mixed at a highly complex level. The characterization of different physical properties in purely geometrical and topological terms, such as quantized transport [2] and electric polarization [3], has lead to the understanding of some undergoing processes governing the physical features of these systems [4]. An important characteristic of geometrical and topological properties in physical systems, arises in their strength against different perturbations. Some examples of those are backscattering in edge states and fault tolerant quantum computation [5 8 ].

Theory of Principal Fiber Bundles (PFB) has also played an important role in quantum mechanics due to its suitability to describe the underlying geometrical and topological properties [9, 10]. Its relation with condensed matter was pointed out by Simon [11], and the existence of an universal PFB [12, 13], which is a purely geometric object, shows that non-adiabatic processes can be used for practical purposes in a similar way than their adiabatic partners.

The interplay between geometry and ac-fields is a very promising field with a huge activity in the last years. The tuning of geometrical properties by the application of ac-fields offers a very interesting scenario, where non-equilibrium systems show quantum phase transitions, and the possibility to create topologically protected states 14 17].

As Berry demonstrated, the phase acquired during the cyclic evolution of a general quantum system can be expressed as a combination of two terms, a dynamical phase $\gamma_{D}$ and a geometric phase $\gamma_{G}$, being the latter obtained due to the parallel transportation of the vector state through the base manifold[18]. The parallel transportation is obtained by means of the 1 -form connection $A^{n}:=i\langle n|d| n\rangle$ ( $d$ is the exterior derivative operator, and $|n\rangle$ is the instantaneous eigenstate), and the geometric phase acquired is given by $\gamma_{G}^{n}=\int_{C} A^{n}$, that only depends on the path followed in the parameter space.

These geometric quantities describe the evolution of the system as the external parameters are varied, but other quantities, such us Chern numbers (topological invariants) can also be defined through the 1 -form $A^{n}$, characterizing the topological properties of the whole base manifold.

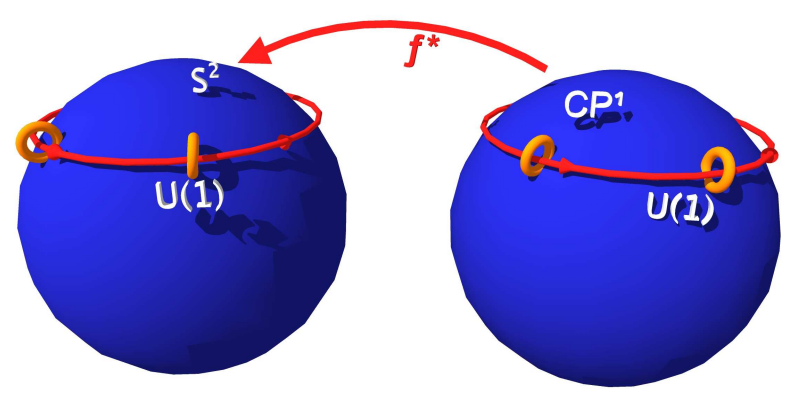

Figure 1: Schematic figure for the relation between adiabatic and non-adiabatic regimes. The function $f^{*}$ maps curves $\mathcal{C}$ from the Bloch sphere (projective Hilbert $\mathbb{C} P^{N-1}$ ) into curves $C$ in the parameter space $\mathcal{S}^{2}=\{\theta, \varphi\}$. The $U(1)$ fibers attached to each point due to the phase invariance of quantum mechanics are represented by small circles. We consider $\mathbb{C} P^{N-1}$ with $N=2$ for the schematic figure (i.e.. $\mathbb{C} P^{1}$ which is the Bloch sphere for a single qubit).

In the present work we consider an extension of the classical model considered by Berry [1], including the spatial degree of freedom, inhomogeneous magnetic fields and non adiabatic evolution. The analytical results show that the interplay between the spin and spatial degree of freedom is remarkably relevant when the ac-field varies between different sites. We calculate the geometric phase and Chern number for both adiabatic and non adiabatic evolution. This model can be realized in different experimental setups, and the results can be easily extended to 
larger size systems such as atomic arrays with a pseudospin degree of freedom. We demonstrate that adiabatic evolution for the ac-field allows tunable geometric phases by varying the external field parameters: $B$ (intensity of the external field) and $\phi$ (phase difference between sites). We show that the variation of the phase difference $\phi$, which introduces the field anisotropy, strongly modifies the Berry phase and the topological invariants. Also that non-adiabatic processes increase the tuning possibilities with the addition of new topological phases, which only appear out of the adiabatic regime.

Model: The system contains a qubit tunneling between two sites $(L, R)$ in presence of and ac-field:

$$
\begin{aligned}
H(t)= & \sum_{i=L, R} B \cos (\theta) S_{z}^{i}+\sum_{\sigma, i \neq j} t_{L R} c_{\sigma, i}^{\dagger} c_{\sigma, j}+ \\
& \sum_{i=L, R} B \sin (\theta)\left[\cos \left(\varphi_{i}(t)\right) S_{x}^{i}+\sin \left(\varphi_{i}(t)\right) S_{y}^{i}\right] .
\end{aligned}
$$

The coupling between sites is given by $t_{L R}, B_{z}$ is the static field which splits the qubit levels, and the ac-field $\vec{B}_{a c}(t)$ couples the different states of the qubit (perpendicular to $B_{z}$ ). In this Hamiltonian, the field has been parametrized according to the angles of a 2-sphere $\mathcal{S}^{2}=$ $\{\theta, \varphi\}$, being $B_{z} \equiv B \cos (\theta), B_{a c}^{x} \equiv B \sin (\theta) \cos \left(\varphi_{i}(t)\right)$ and $B_{a c}^{y} \equiv B \sin (\theta) \sin \left(\varphi_{i}(t)\right)$. This is a very appropriate parameter space for a circularly polarized ac field with time dependence $\varphi_{i}(t)=\Omega t+\phi_{i}$, which is the case considered in this work. For simplicity, we consider symmetrical $B_{z}$ and $B_{a c}(t)$ at each site.

For this Hamiltonian we calculate the geometric and topological properties by means of the connection 1-form $A^{n}$. We consider both, the adiabatic and non-adiabatic time evolution for phase differences $\phi=\phi_{L}-\phi_{R} \in\{0, \pi\}$, i.e. magnetic fields in phase or in phase opposition respectively. We show that in the last case, interesting topological properties arise. Furthermore, we calculate the phase diagram demonstrating that non-adiabatic evolution leads to the appearance of new topological phases.

Adiabatic case: The adiabatic case considers slow time evolution. This allows to define instantaneous eigenstates and neglect the transitions to other energy levels. The instantaneous energies $E_{n}(\phi)$ can be characterized by two indexes after the diagonalization $n \equiv$ $\left(m_{1}= \pm 1, m_{2}= \pm 1\right)$ :

$$
\begin{aligned}
& E_{n}(0)=-\frac{B}{2}\left(m_{1}+m_{2} \lambda\right) \\
& E_{n}(\pi)=-\frac{m_{1} B}{2} \sqrt{1+\lambda^{2}-2 m_{2} \lambda \cos (\theta)},
\end{aligned}
$$

being $\lambda=2 t_{L R} / B$. We can see that both instantaneous energies are $\varphi$ independent. The case $\phi=0$ reflects the classical result obtained by Berry (i.e.. without $\theta$ dependence), with an extra splitting due to the tunneling $t_{L R}$. However, the case $\phi=\pi$ shows a $\theta$ dependence which has not been obtained previously. That is, the instantaneous energies now depend on the ratio between the ac-field and the Zeeman splitting through $\theta$ (Fig/2), and not just on the total intensity $B=|\vec{B}|=\sqrt{B_{a c}^{2}+B_{z}^{2}}$.

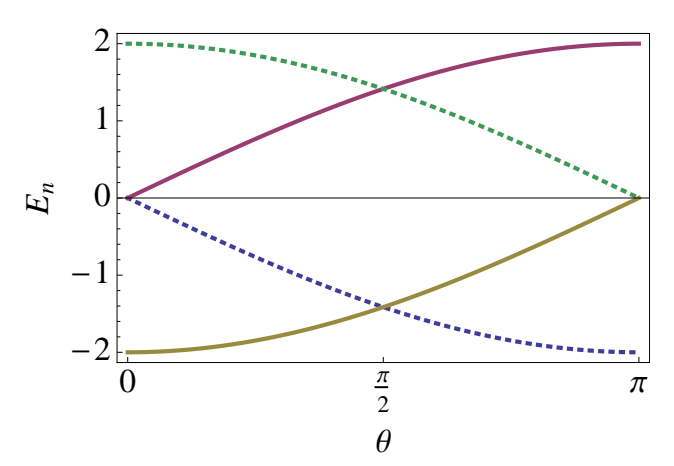

Figure 2: Instantaneous energies $E_{n}(\pi)$ vs $\theta$ for $t_{L R}=1$, $\phi=\pi$ and $B=2$ (dashed lines: $E_{ \pm, \pm}(\pi)$ and continuous lines: $\left.E_{\mp, \pm}(\pi)\right)$.

In the calculation of the instantaneous eigenstates, due to the parameter space which is a 2-sphere, we need to consider two different charts. This is due to the fact that the surface of a sphere cannot be directly mapped to a plane. The transformation of the instantaneous eigenstates between different charts is given by phase factors [10]. This is a signature of non-trivial geometrical and topological properties. However, we shall not worry about this, because we are interested in the geometric phase and the curvature tensor $F^{n}=d A^{n}$ (needed for the calculation of the Chern number), which are globally defined for the case of Abelian theories.

In order to obtain the geometric phase, we calculate, using the instantaneous eigenvectors, the connection 1form $\mathbf{A}^{n}=\left(A_{\varphi}^{n}, A_{\theta}^{n}\right)$. The result shows that the phase difference strongly modifies the geometric phase (Fig 3), which is $t_{L R}$ dependent for the case of $\phi=\pi$ :

$$
\begin{aligned}
\gamma_{G}^{n}(\phi=0) & =\pi\left(1-m_{1} \cos (\theta)\right) \\
\gamma_{G}^{n}(\phi=\pi) & =\pi \frac{m_{1}\left(\lambda m_{2}-\cos (\theta)\right)+f_{m_{2}}(\lambda, \theta)}{f_{m_{2}}(\lambda, \theta)},
\end{aligned}
$$

being $f_{m_{2}}(\lambda, \theta) \equiv \sqrt{1+\lambda^{2}-2 m_{2} \lambda \cos (\theta)}$. Note that for $\phi=0$ (spatially homogeneous field) $\gamma_{G}^{n}$ does not depend on $t_{L R}$. This fact, implies that it remains invariant for several tunnel coupled two level systems as long as $\phi=0$ (i.e. the field is spatially homogeneous). Once we add the spatial anisotropy through the phase difference $\phi \neq 0$, Berry's result is modified. It adds an interplay between tunneling and ac-field which has not been previously analyzed.

The next step is the calculation of the curvature 2-form $F^{n}=d A^{n}=\left(\frac{\partial}{\partial \varphi} A_{\theta}^{n}-\frac{\partial}{\partial \theta} A_{\varphi}^{n}\right) d \varphi \wedge d \theta$, that will be used 


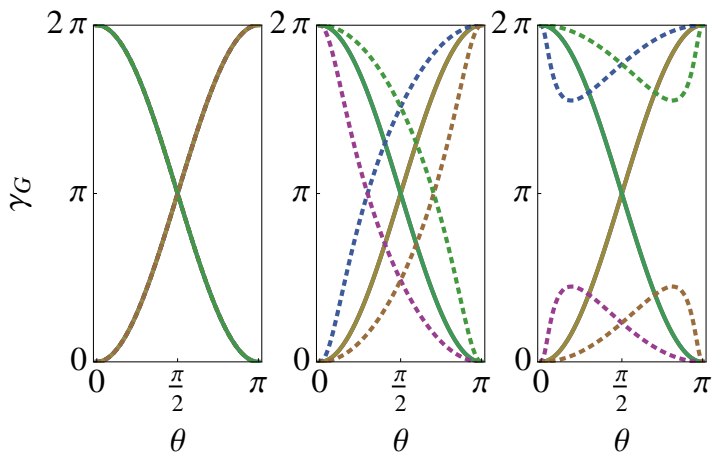

Figure 3: Geometric phase in the adiabatic limit $\gamma_{G}$ vs $\theta$ for different $\lambda(\lambda=0$ (left), $\lambda=0.6$ (center) and $\lambda=1.2$ (right)). Dashed (continuous) lines: $\phi=\pi(\phi=0)$. Note that as $\lambda=2 t_{L R} / B$ is increased, a gap appears for the case $\phi=\pi$. The value at which the topological phase transition occurs is exactly where the gap opens $(\lambda=1)$.

for the calculation of the Chern numbers:

$$
\begin{aligned}
& F_{\phi=0}^{n}=m_{1} \frac{\sin (\theta)}{2} \\
& F_{\phi=\pi}^{n}=m_{1} \frac{\sin (\theta)}{2} \frac{1-\lambda m_{2} \cos (\theta)}{\left(1+\lambda^{2}-2 \lambda m_{2} \cos (\theta)\right)^{3 / 2}} .
\end{aligned}
$$

The result shows that for $\phi=\pi$, the original curvature $\left(\mathrm{Eq}\right.$ 5) is renormalized by a factor which depends on $t_{L R}$ and $m_{2}(\mathrm{Eq}[6)$. For $\lambda=1$, the curvature 2-form inverts its value, and at the limit of large tunneling $t_{L R} \gg B$ the curvature tends to zero for all $\theta$, evolving towards a flat space. The first Chern number is given by:

$$
c_{1}^{n}(\lambda)=\int_{\mathcal{S}^{2}} \frac{F^{n}}{2 \pi}= \begin{cases}m_{1} \Theta(1-\lambda) & \text { for } \phi=\pi \\ m_{1} & \text { for } \phi=0\end{cases}
$$

being $\Theta(x)$ the Heaviside step function. Hence, for $\phi=$ $\pi$, the system undergoes a transition from a topological phase $\mathbb{Z}\left(c_{1} \neq 0\right)$ to a trivial phase $\left(c_{1}=0\right)$ at $\lambda=1$ by tuning the ratio between the hopping $t_{L R}$ and the intensity of the ac-field $|\vec{B}|$.

All results in this section has been obtained under the adiabatic assumption, but this is an approximation for ideal slow evolution. Therefore a natural question arises: How do the previous results change when we consider non-adiabatic evolution?.

Non-adiabatic case: Aharonov and Anandan (A-A) proposed a generalization of the Berry phase to nonadiabatic processes, the A-A phase $\gamma_{\mathrm{A}-\mathrm{A}}$ [18]. As later was pointed out, this generalization corresponds to a universal connection $\mathcal{A}$ in a universal PFB $\mathbb{C} P^{\infty}$ or $\mathrm{A}$ A bundle 19]. It establishes a general relation between the classification of $U$ (1) PFB and quantum mechanical systems [13]. The relation between Berry's and Anadan's result is stablished through a function $f_{n}^{*}$ (pullback bundle), that maps curves $\mathcal{C}$ defined in $\mathbb{C} P^{\infty}$ to curves $C$ in the parameter space, i.e. $C=f_{n}^{*}(\mathcal{C})$, then $f_{n}^{*}$ has all the information for the computation of the geometric phase (the explicit form of the Hamiltonian determines $f_{n}^{*}$ ).

We will show below that for the non-adiabatic case, the geometric phase $\gamma_{\mathrm{A}-\mathrm{A}}$ and the phase diagram match the previous results in the adiabatic limit 20.

Considering the present setup in the non-adiabatic regime, we shall demonstrate that it can be exactly solved (without the adiabatic approximation), and calculate the geometric phase $\gamma_{\mathrm{A}-\mathrm{A}}$ and the topological invariants.

By means of a unitary transformation to a co-rotating frame with the ac-field, we obtain a static Hamiltonian which can be diagonalized. The unitary transformation is given by $U(t)=\exp \left\{-i \Omega t\left(S_{z}^{L}+S_{z}^{R}\right)\right\}$, and the transformed Hamiltonian $\tilde{H}=U^{\dagger} H U-i U^{\dagger} \dot{U}$ reads:

$$
\begin{aligned}
\tilde{H}= & \sum_{i=L, R}(B \cos (\theta)-\Omega) S_{z}^{i}+\sum_{\sigma, i \neq j} t_{L R} c_{\sigma, i}^{\dagger} c_{\sigma, j} \\
& +\sum_{i=L, R} B \sin (\theta)\left[\cos \left(\phi_{i}\right) S_{x}^{i}+\sin \left(\phi_{i}\right) S_{y}^{i}\right] .
\end{aligned}
$$

which is independent of $t$. Note that the time dependence of the transformation renormalizes the energy levels by $\Omega$.

The diagonalization of the Hamiltonian leads to the energies $\mathcal{E}_{n}(\phi)$ :

$$
\begin{aligned}
& \mathcal{E}_{n}(0)=-m_{1} \frac{B}{2} \sqrt{1+\mu^{2}-2 \mu \cos (\theta)}-m_{2} t_{L R} \\
& \mathcal{E}_{n}(\pi)=-m_{1} \frac{B}{2} \sqrt{1+\Delta_{m_{2}}^{2}-2 \Delta_{m_{2}} \cos (\theta)}
\end{aligned}
$$

being $\mu \equiv \Omega / B$ and $\Delta_{m_{2}} \equiv\left(\Omega+2 m_{2} t_{L R}\right) / B$. Note that in both cases, the limit $\Omega \rightarrow 0$ matches the adiabatic result. Note also the similarities between Eq2 and Eq 9 , where the frequency renormalizes the different parameters.

Interestingly, the energies present degeneracy points for both $\phi=0, \pi$. Our calculations show that the geometric phases are also Abelian at degeneracy points due to the structure of the Floquet operator, which is in agreement with the results of [21].

The solutions to the Schrdinger equation are given by the transformation of the eigenvectors $|\tilde{\psi}\rangle$ obtained by diagonalizing $\mathrm{Eq} 8$ to the original frame, i.e. $|\psi(t)\rangle=$ $U(\varphi(t))|\tilde{\psi}\rangle$. Therefore, the calculation of the 1-form $\mathcal{A}_{\varphi}$ gives:

$$
\mathcal{A}_{\varphi}=i\left\langle\psi(t)\left|\partial_{\varphi}\right| \psi(t)\right\rangle d \varphi=\left\langle\tilde{\psi}\left|S_{z}\right| \tilde{\psi}\right\rangle d \varphi
$$

being the A-A geometric phase $\gamma_{\mathrm{A}-\mathrm{A}}=\oint_{\mathcal{C}} \mathcal{A}_{\varphi}=2 \pi \mathcal{A}_{\varphi}$, i.e. :

$$
\begin{aligned}
& \gamma_{\mathrm{A}-\mathrm{A}}^{n}(0)=m_{1} \pi \frac{(\mu-\cos (\theta))}{\sqrt{1+\mu^{2}-2 \mu \cos (\theta)}} \\
& \gamma_{\mathrm{A}-\mathrm{A}}^{n}(\pi)=m_{1} \pi \frac{\left(\Delta_{m_{2}}-\cos (\theta)\right)}{\sqrt{1+\Delta_{m_{2}}^{2}-2 \Delta_{m_{2}} \cos (\theta)}}
\end{aligned}
$$


and the curvature:

$$
\begin{aligned}
& F^{n}(0)=m_{1} \frac{\sin (\theta)}{2} \frac{1-\mu \cos (\theta)}{\left(1+\mu^{2}-2 \mu \cos (\theta)\right)^{3 / 2}} \\
& F^{n}(\pi)=m_{1} \frac{\sin (\theta)}{2} \frac{\left(1-\Delta_{m_{2}} \cos (\theta)\right)}{\left(1+\Delta_{m_{2}}^{2}-2 \Delta_{m_{2}} \cos (\theta)\right)^{3 / 2}}
\end{aligned}
$$

We can see that the geometric properties for $\phi=0$ does not depend on $t_{L R}$ nor on $m_{2}$ (always a degeneracy for states with different $m_{2}$ is present). By contrary, the $\phi=\pi$ case presents different curvature for each state, but because of the functions $\Delta_{ \pm}$cannot be independently modified by changing the system parameters $B, \Omega$ and $t_{L R}$, the geometric phases and curvatures also cannot. The Chern numbers can be calculated through the curvature, and then, obtain the phase diagrams as a function of $\Delta_{m_{2}}$ or $\mu$ :

$$
\begin{aligned}
c_{1}^{n}(0) & =\frac{m_{1}}{2}(1+\operatorname{sign}(1-\mu)) \\
c_{1}^{n}(\pi) & =m_{1} \Theta\left(1-\left|\Delta_{m_{2}}\right|\right) \\
& =m_{1} \Theta\left(1-\left|\Omega+2 m_{2} t_{L R}\right| / B\right)
\end{aligned}
$$

The results obtained for the geometric phase show certain interesting features. For the $\phi=0$ case, the non-adiabatic phases $\gamma_{\mathrm{A}-\mathrm{A}}$ present some corrections in $\mu$ (which is precisely the adiabatic parameter) to the adiabatic case, but they remain degenerate in pairs, and the variation of $\mu$ modifies all geometric phases simultaneously. Also we must note that $\gamma_{\mathrm{A}-\mathrm{A}}^{n}(\phi=0)(\mathrm{Eq}[10)$, as in the adiabatic case (Eq 3 , does not depend on $m_{2}$ nor on $t_{L R}$, meaning that systems with a larger number of coupled spins would have the same geometric phase.

Regarding the phase diagram, the $\phi=0$ case just contains two phases: The topologically trivial and the $\mathbb{Z}$ phase. The trivial phase appears when we go out of the adiabatic regime by increasing the frequency $(\mu=$ $\Omega / B>1$ ), while the $\mathbb{Z}$ phase governs the whole adiabatic regime.

Concerning the $\phi=\pi$ case in the non-adiabatic regime, the geometric phases (Eq11) depend on two functions $\Delta_{m_{2}}$, and hence on both indexes $\left(m_{1}, m_{2}\right)$ of the state vector. This allows to modify the geometric and topological properties differently for each state. This is an important difference with the $\phi=0$ case, because now the geometric phase for each state behaves differently when we vary $t_{L R}, \Omega$ and $B$ for $\phi=\pi$. This is reflected in the phase diagram, where two new topological phases appear: $(0, \mathbb{Z})$ and $(\mathbb{Z}, 0)$ (first index labels states with $m_{2}=+1$ and second index states with $m_{2}=-1$ ) in which only two of the states have non-vanishing Chern number. More interestingly, at arbitrary high frequencies $\Omega$ we show that states with non-vanishing Chern number can appear (Fig 4).

Because the functions $\Delta_{ \pm}$depend both on the system parameters, we plot the phase diagram as a function of
$B$ and $\Omega$ for fixed $t_{L R}$. Interestingly, the $(\mathbb{Z}, 0)$ phase is not accessible (Fig 4). This is due to dependence on $B$, $t_{L R}$ and $\Omega$ of the functions $\Delta_{ \pm}$. Note that for a different dependence on the parameters, the $(\mathbb{Z}, 0)$ phase could appear. We also observed that the variation of the parameter $t_{L R}$ only modifies the width of the $(0, \mathbb{Z})$ phase, such that in the limit $t_{L R} \rightarrow 0$ we obtain the phase diagram for the $\phi=0$ case.

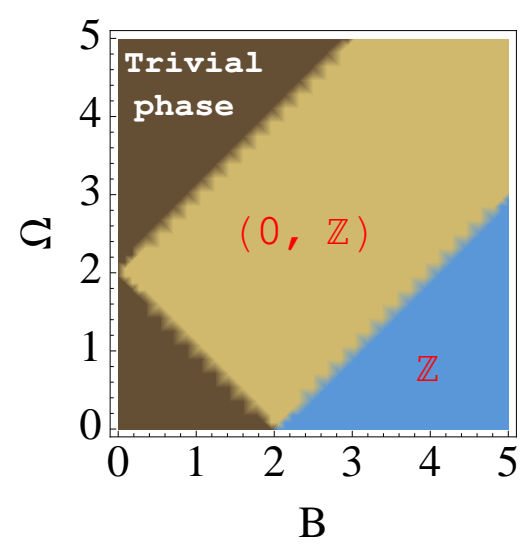

Figure 4: Topological phases diagram vs $(B, \Omega)$ for fixed $t_{L R}=1$ and $\phi=\pi$. The phase diagram shows the physically accessible topological regions. Note that the adiabatic limit is found by choosing $\Omega=0$ with the trivial and fully topological phases described above. In addition, for non adiabatic processes (i.e. $\Omega>0$ ) a new phase $(0, \mathbb{Z})$ appears, in which just half of the states are characterized by a non vanishing Chern number.

The existence of phases with non-vanishing Chern number implies a non-trivial bundle, and therfore holonomy elements different from the identity. It means that it is impossible to avoid the geometric phases in the study of these systems. The geometric phases are local, in difference with the Chern numbers, and can be different from zero even for regions where $c_{1}=0$. Our results can be important for experiments involving the measurement of geometric phases, which can be performed by using superconducting qubits and quantum state tomography 22$]$ among other setups 23.

Summary: Summarizing, we analyzed the geometric phases and topology of tunnel coupled systems with an on-site pseudo-spin degree of freedom, and driven by acfields. We have considered the case of an electron spin, tunneling between two different sites, which is coupled to an ac magnetic field that produces transitions between the spin up/down levels. The interplay between the spatial and spin degree of freedom due to the ac magnetic field, as well as its dependence with the external field parameters $B, \Omega$ and $\phi$ (phase difference between different sites) is analyzed from a geometrical point of view.

Interestingly, we found that a system presenting spatial anisotropy (in our case due to the phase difference $\phi$ between the external ac driving fields in different sites) leads 
to a complex and rich behavior compared with Berry's classical result for a localized spin in the adiabatic regime. Our analysis is also extended to the non-adiabatic regime. In this case we find geometrical phases which depend in a non trivial way on the spatial field anisotropy. The results generalize the well known Aharonov-Anandan phase for localized spins, resulting in a novel topological phase diagram (Fig 4). Our results are the basis for the analysis of larger systems with spatial periodicity which will be the subject of a future work.

Although the topological phase transition can be difficult to be directly measured in our setup, the measurement of geometric phases could indirectly show the topological phase transition (see Fig 3). Setups involving quantum dots in slanting magnetic fields [24] and quantum circuits 22] can be used for this purpose. Also, the non-adiabatic analysis shows how the topological invariants change out of the adiabatic regime, leading to possible applications in non-adiabatic quantum computation.

We acknowledge MAT 2011-24331 and ITN, grant 234970 (EU) for financial support. A. Gómez-León acknowledges JAE program.

[1] M. Berry, Proc. Roy. Soc. London, Ser. A , 45 (1984).

[2] D. Thouless, Physical Review B 27, 6083 (1983).

[3] R. King-Smith, Phys. Rev. B 47, 1651 (1993).

[4] D. Xiao and M. Chang, Reviews of Modern Physics (2010), arXiv:arXiv:0907.2021v1.

[5] M. König, S. Wiedmann, C. Brüne, A. Roth, H. Buhmann, L. W. Molenkamp, X.-L. Qi, and S.-C. Zhang, Science 318, 766 (2007).

[6] A. Kitaev, Annals of Physics 303, 2 (2003), arXiv:9707021v1 [arXiv:quant-ph].

[7] E. Dennis, A. Kitaev, A. Landahl, and J. Preskill,
Journal of Mathematical Physics 43, 4452 (2002), arXiv:0110143v1 [arXiv:quant-ph].

[8] P. Zanardi, Physics Letters A , 94 (1999).

[9] T. T. Wu and C. N. Yang, Phys. Rev. D 12, 3845 (1975).

[10] A. Bohm, A. Mostafazadeh, H. Koizumi, Q. Niu, and J. Zwanziger, The Geometric Phase in Quantum Systems (Springer, 2003).

[11] B. Simon, Physical Review Letters 51, 2167 (1983).

[12] J. Anandan and L. Stodolsky, Physical Review D 35, 2597 (1987).

[13] A. Bohm, L. Boya, A. Mostafazadeh, and G. Rudolph, Journal of Geometry and Physics 12, 13 (1993).

[14] N. Lindner, G. Refael, and V. Galitski, Nature Physics 7, 490 (2011), arXiv:arXiv:1008.1792v1.

[15] T. Kitagawa, E. Berg, M. Rudner, and E. Demler, Physical Review B 82, 235114 (2010), arXiv:arXiv:1010.6126v1.

[16] V. Bastidas, C. Emary, B. Regler, and T. Brandes, Physical Review Letters 108, 043003 (2012).

[17] M. Tomka, A. Polkovnikov, and V. Gritsev, Physical Review Letters 108, 080404 (2012).

[18] Y. Aharonov and J. Anandan, Physical Review Letters 58, 1593 (1987).

[19] $\mathbb{C} P^{N}$ is the complex projective space of $\mathrm{N}$ dimension. This is the set of equivalence classes $\mathrm{P}(\mathrm{H})$ for states $|\psi\rangle \in$ $H$, such that the equivalence relation is given by $|\psi\rangle \sim$ $c|\psi\rangle$, being $\mathrm{H}$ the Hilbert space of dimension $\mathrm{N}$ and $\mathrm{c}$ a complex number .

[20] Note that the Aharonov-Anandan PFB is a fully geometric entity and does not assume any approximation such us adiabaticity .

[21] A. Mostafazadeh, J. Phys. A: Math. Gen. 31, 9975 (1998).

[22] P. J. Leek, J. M. Fink, A. Blais, R. Bianchetti, M. Göppl, J. M. Gambetta, D. I. Schuster, L. Frunzio, R. J. Schoelkopf, and A. Wallraff, Science 318, 1889 (2007).

[23] F. Nagasawa, J. Takagi, Y. Kunihashi, M. Kohda, and J. Nitta, Phys. Rev. Lett. 108, 086801 (2012).

[24] M. Pioro-Ladrière, T. Obata, Y. Tokura, Y.-S. Shin, T. Kubo, K. Yoshida, T. Taniyama, and S. Tarucha, Nature Physics 4, 776 (2008). 